\title{
CONTRIBUTION OF MITOCHONDRIAL DISFUNCTION IN THE DEVELOPMENT OF SPORADIC ALZHEIMER'S DISEASE: EVIDENCE FROM OXYS RATS
}

\author{
Mikhail A. Tyumentsev ${ }^{1}$, Natalia A. Muraleva ${ }^{1}$, \\ Valentin A. Vavilin ${ }^{2}$, Natalia A. Stefanova ${ }^{1}$, Nataliya G. Kolosova ${ }^{1}$ \\ ${ }^{1}$ Institute of Cytology and Genetics, Novosibirsk, e-mail: kolosova@bionet.nsc.ru \\ ${ }^{2}$ Institute of Molecular Biology and Biophysics, Novosibirsk
}

Many lines of evidence suggest that decreased mitochondrialfunction is a hallmark of aging and that mitochondrialdysfunction has a central role in Alzheimer's disease (AD), the most common type of age-related dementia worldwide, which has no cure. The central role of mitochondria in aging was first proposed by Denham Harman, on the basis of his original theory that aging is caused by the accumulation of damage resulting from reactive oxygen species (ROS) (Harman, 2009). However, recent findings suggest that formation of ROS is neither the primary nor the initial cause of aging. Moreover, transient stress on mitochondria, including mitochondrial ROS (playing a critical role in a number of intra- and extracellular processes), elicits beneficial changes that extend the lifespan (Rose et al., 2017). Clearly, mitochondrial function regulates the rate of aging, and mitochondrial dysfunction takes the center stage in the pathophysiology of age-related neurodegenerative disorders, but the underlying mechanisms remain unclear. We explored the role of mitochondrial dysfunction in the development of sporadic late-onset $\mathrm{AD}$, which accounts for $95 \%$ of all disease cases, using senescence-accelerated OXYS rats. This rat model simulates key characteristics of AD including tau protein hyper-phosphorylation, synaptic losses, neuronal cell death, behavioral alterations, and a decrease in cognitive functions on the background of increase in APP levels, enhanced accumulation of $A \beta$, and formation of amyloid plaques in the brain. The genome of OXYS rats lacking the mutations in the App, Psen1, and Psen2 genes, which are specific for the early form of $\mathrm{AD}$, also speaks in favor of the this model matching the particular criteria of sporadic AD (Stefanova et al., 2014). According to our data, the development and progression of AD-like pathology in OXYS rats may be caused by mitochondrial dysfunction (Stefanova et al., 2014-2016, Kolosova et al., 2017, Tyumentsev et al., 2018). Here we summarize our evidence supporting the validity of this assertion.

First, already at the age of 20 days, i.e., at the preclinical stage of the development of AD-like pathology, OXYS rats showed some characteristic changes in hippocampal mitochondria, which increased in size during the manifestation (the age of 5 months) and progression of these pathological changes (18 months). Even at this early age, OXYS rats show significantly lower respiratory complex IV activity and a tendency toward a decrease in the activity of complexes I and V. The persistent depression of respiratory chain activity in the hippocampal mitochondria of OXYS rats is observed throughout the lifespan. Simultaneously this decreased activity, OXYS rats show increased fusion, which leads to formation of larger mitochondria. Such fused highly integrated mitochondrial phenotype is thought to be geared toward upregulation of energy supply via ATP synthesis. Therefore, we did not detect any signs of energy deficiency in OXYS rats' brain up to the age of 3 months, when we estimated the brain energy metabolism by 31P NMR spectroscopy. What's more, we have previously detected an increased phosphate potential in 2- to 3-week-old OXYS rats compared to Wistar rats (elevated creatine phosphateto inorganic phosphate $(\mathrm{PCr} / \mathrm{Pi})$ and $(\mathrm{PCr} / \mathrm{ATP})$ ratios) which could be a consequence of adaptation of the brain to hypoxic conditions (Sergeeva et al., 2006). Later, we have shown that the prevalence of the mitochondrial common deletion (4834 bp in mtDNA; which is the most typical form of mtDNA oxidative damage associated with aging and degenerative diseases) in the hippocampus of OXYS rats is increased, especially at the stage of completion of brain development in the postnatal period (Loshenova et al., 2015). 
This level remained elevated not only at the stages preceding the manifestation and the development of the signs of $\mathrm{AD}$ but also during their progression.

By the age of 20 days, final maturation of the brain takes place, during which many brain regions, including the hippocampus, undergo development and histogenesis associated with the formation of interneuronal contacts and elimination of "transitional" cell populations by apoptosis. High apoptotic activity and, according to the latest data, neurogenesis, point to enhanced ROS production. It is noteworthy that we found that early age (20 days) is the only period when brain mitochondria from OXYS rats show signs of increased production of ROS. It should be noted that increased production of ROS occurs much earlier than amyloid plaque accumulation, tau hyperphosphorylation, or behavioral abnormalities in OXYS rats (Stefanova et al., 2015). For a long time overproduction of ROS has been regarded as the cornerstone of mitochondrial pathology and of neurodegenerative diseases in which mitochondrial pathology plays an important part (Srivastava, 2017). In contrast, we did not see enhanced formation of ROS by mitochondria isolated from the brain of 5- and 24-month-old rats when the ADlike pathology developed and progressed and well-pronounced structural disturbances in mitochondria were revealed in the hippocampus of OXYS rats by electron microscopy. We hypothesized that the state of mitochondria in OXYS rats may be characterized as mild uncoupling, which can decrease ROS production by lowering the membrane potential; this change increases the rate of electron transfer and oxygen consumption while maintaining respiratory complexes in a more oxidized state (Korshunov et al., 1997).

Second, according data of RNA-seq (Stefanova et al., 2015) the expression profile of mitochondrial genes in the brain of OXYS rats is significantly different from that of normal control rats, including the possible mitochondrial energy deficiency.Progression occurs against the background of alteration in the cortex of the genes associated with the metabolic pathway of $\mathrm{AD}$, including those related to processing of the precursor protein $\mathrm{A} \beta$, aggregation and degradation of $A \beta$, synaptic processes, and mitochondrial dysfunction. Our results are consistent with RNA-Seq data on changes in the transcriptome of the prefrontal cortex of patients with AD.

Data on the change of mitochondrial mass during brain aging are limited but overall suggest that with advancing age, this parameter increases or tends to increase (Swerdlow, 2011). In 2-year-old Wistar rats, we observed a tendency toward an increase in the number of mitochondria in hippocampal neurons. Furthermore, in OXYS rats, their amount decreased by age 5 months, remained at this level at 24 months and was significantly lower than that in Wistar rats. Starting at the age of 4 months and until age 24 months, we observed a shift from mitochondrial fusion toward fission as evidenced by increasing DRP1 content. Decreased mitochondrial fusion and formation of a fragmented mitochondrial phenotype is a characteristic sign of many models of neurodegenerative diseases. Mitochondrial fission itself may also contribute to the removal of mitochondria through subsequent mitophagy contributing to the diminishing number of mitochondria. Overall, the observed events appear to signify a mismatch between the intensities of biogenesis and mitophagy; this phenomenon as a whole is also considered a hallmark of age-associated neurodegenerative diseases.

Mitochondria are physically and biochemically in contact with ER via formation of mitochondria-associated membranes harboring various effector and signaling proteins positioned in lipid rafts. In addition to mitochondrial dynamics, ER contact sites play a pivotal role in mitophagy, membrane lipid transport, and calcium signaling. We showed increased formation of ER contact sites on neuronal mitochondria of OXYS rats during manifestation of AD signs (age 5 months) that may be a part of an ongoing compensatory reaction aimed at increasing the supply of ATP and improving the quality control of the remaining mitochondria. Similarly, OXYS neuronal mitochondria starting from a young age are more frequently positioned next to the nucleus, with the frequency increasing threefold during the rats' lifespan, suggesting that starting at the age of the first signs of AD, the demand for ATP in the neuronal nucleus increases. Notably, in OXYS rats, formation of ER contact sites after an increase at the age of 5 months underwent a significant decrease later, at the age of active progression of 
AD-like pathology. This change in ER contact sites is accompanied by signs of $A D$, such as $A \beta$ accumulation, tau hyperphosphorylation, synaptic impairment, and neuronal death. These data suggest that by the late age (24 months), an initial compensatory response aimed against A $\beta$ accumulation no longer functions. Additionally, at this age, OXYS rats accumulate more severely damaged mitochondria as compared to Wistar rats; this finding indicates that OXYS mitochondria are under a significant workload, where quality control mechanisms become insufficient.

Summarizing the results of our studies, we compared the age-dependent changes in neuronal mitochondria with the development of AD signs in OXYS rats. We can see that already at the age of 20 days in the absence of any signs of AD, OXYS rats show decreased activity of complex IV of the respiratory chain, increased ROS production, and accumulation of deletions in mitochondrial DNA. Manifestation of behavioral aberrations and deterioration of cognitive abilities in OXYS rats take place at the age of 3-4 months during the increase in mitochondrial aberrations and hyperphosphorylation of tau protein. Increased accumulation of $\mathrm{A} \beta$ in the brain of OXYS rats occurs at age 7-12 months, concurrently with well-pronounced neurodegenerative changes, and becomes a secondary event in relation to mitochondrial dysfunction. The key role of mitochondrial dysfunction in the pathophysiology of AD is confirmed by the ability of mitochondria-targeted antioxidant SkQ1 to alleviate the neurodegenerative alterations via improvement of structural and functional state of mitochondria in OXYS rats. Namely, SkQ1 prevents the neuronal loss and synaptic damage, enhances neurotrophic supply, and decreases the amounts of toxic forms of $A \beta$ and tau hyperphosphorylation in the hippocampus of OXYS rats, thereby resulting in improvement of cognitive function (Stefanova et al., 2014, 2016; Kolosova et al., 2017). Collectively, these data allow us to conclude that mitochondrial dysfunction appears at least to mediate or possibly even initiate pathological molecular cascades of AD-like pathology in OXYS rats. Therefore, mitochondrial dysfunction may be considered a predictor of the early development of the late-onset form of AD in humans. If so, mitochondrial dysfunction actually constitutes a mechanistic link between brain aging and $\mathrm{AD}$.

\section{References}

Harman D. Origin and evolution of the free radical theory of aging: A brief personal history, 1954-2009 // Biogerontology. - 2009. - Vol. 10, N 6. - P. 773-781.

Kolosova N.G. et al. Antioxidant SkQ1 Alleviates Signs of Alzheimer's Disease-like Pathology in Old OXYS Rats by Reversing Mitochondrial Deterioration // Curr. Alzheimer Res. - 2017. Vol. 14, N 12. - P. 1283-1292.

Korshunov S.S., Skulachev V.P., Starkov A.A. High protonic potential actuates a mechanism of production of reactive oxygen species in mitochondria // FEBS Lett. Federation of European Biochemical Societies. - 1997. - Vol. 416, N 1. - P. 15-18.

Loshchenova P.S. et al. Influence of antioxidant SkQ1 on accumulation of mitochondrial DNA deletions in the hippocampus of senescence-accelerated OXYS rats // Biochem. - 2015. - Vol. 80, N 5. - P. 596-603.

Rose G., Santoro A., Salvioli S. Mitochondria and mitochondria-induced signalling molecules as longevity determinants // Mech. Ageing Dev. Elsevier Ireland Ltd, 2017. - Vol. 165. - P. 115-128.

Sergeeva et al., 2006.

Stefanova N.A. et al. Amyloid accumulation is a late event in sporadic Alzheimer's disease-like pathology in nontransgenic rats // Oncotarget. - 2015. - Vol. 6, N 3. - P. 1396-1413.

Stefanova N.A. et al. Senescence-accelerated OXYS rats: A model of age-related cognitive decline with relevance to abnormalities in Alzheimer disease // Cell Cycle. - 2014. - Vol. 13, N 6. P. 898-909.

Stefanova N.A. et al. An antioxidant specifically targeting mitochondria delays progression of Alzheimer's disease-like pathology // Aging (Albany. NY). - 2016. - Vol. 8, N 11. - P. 2713-2733.

Stefanova N.A. et al. Alzheimer's disease-like pathology in senescence-accelerated OXYS rats can be partially retarded with mitochondria-targeted antioxidant SkQ1 // J. Alzheimer's Dis. - 2014. Vol. 38, N 3. - P. 681-694.

Tyumentsev M.A., Stefanova N.A., Muraleva N.A., RumyantsevaY.V., Kiseleva E., Vavilin V.A., Kolosova N.A. Mitochondrial dysfunction as a predictor and driver of Alzheimer's disease-like pathology in OXYS rats // J. Alzheimer's Dis. - 2018. - Vol. 63, N 3. 